\title{
Sistematização das melhores práticas de desenvolvimento de produtos para acesso livre e compartilhamento na internet
}

\author{
Creusa Sayuri Tahara Amaral, Dr. \\ EESC - USP

\section{Henrique Rozenfeld} \\ Departamento de Engenharia de Produção - Escola de Engenharia de São Carlos - USP
}

\begin{abstract}
Uma das formas de melhorar a eficiência no processo de desenvolvimento de produto (PDP) é por meio da criação de padrões, denominados de modelo de referência, que descrevem as atividades envolvidas no processo, estabelecendo uma linguagem comum a todos envolvidos no processo. Existe uma dificuldade em fazer destas melhores práticas uma realidade, principalmente para as pequenas e médias empresas (PME's). Propõe-se, neste trabalho, uma ferramenta na web que sistematiza as melhores práticas em PDP, colocando-as à disposição da comunidade em geral, especialmente profissionais de PME's e educadores. Essa ferramenta foi baseada no modelo de referência unificado, proposto por Rozenfeld et al. (2006). Como resultado, discute-se a melhor forma de se organizar modelos de referência na web e a experiência na sua construção.
\end{abstract}

Palavras-chave: Modelo de referência, Processo de desenvolvimento de produtos, melhores práticas

To maintain their competitiveness in the market the companies should invest in the PDP systematization which provides a pattern, usually denominated as reference model. It describes company's PDP establishing a common language to the people get involved in the product development. Even so, there is a lack in doing a reality of these best practices, mainly for the small and medium companies. This paper describes an internet free access tool that systematize those best practices in the internet for professionals and academic people, according the unified model proposed by ROZENFELD et al. (2006). As result the papers discuss how to organize and present reference models in the internet and this creation experience.

Keywords: Reference Model, Product development process, best practice

\section{Introdução}

A internacionalização tem provocado mudanças na economia mundial e modificou o perfil do mercado nacional. A entrada de produtos importados elevou os padrões de atendimento ao cliente, com produtos eficientes, inovadores, com alta qualidade e preço baixo. As empresas que desejarem se manter competitivas nesse cenário devem, entre outros fatores, investir em aprimorar e incrementar o processo de desenvolvimento de produto (PDP), para torná-lo eficaz e sistematicamente planejado, com o objetivo de atender de modo mais ágil às novas necessidades do mercado, oferecendo produtos inovadores e mais competitivos.

De modo geral, ter um PDP bem estruturado pode resultar em: redução no lead-time de desenvolvimento, repetibilidade dos projetos de desenvolvimento, maior racionalização no uso das informações, maior facilidade para treinar novas pessoas no processo e reutilização de conhecimentos gerados em outros projetos. Estruturar o PDP significa dotar a empresa de um padrão a ser seguido pelos diferentes times de desenvolvimento no planejamento e realização dos projetos de desenvolvimento de produtos. Este padrão pode ser denominado também de modelo de referência, que descreve o PDP da empresa, estabelecendo uma linguagem comum a todos envolvidos no 
desenvolvimento de produto. Nele podem ser representadas as atividades de um processo, assim como os métodos, ferramentas, dados de entrada/saída. Todas essas informações de um modelo de referência podem ser consideradas como melhores práticas que podem ser adotadas pelas empresas na melhoria de seu PDP.

Porém, existe uma dificuldade em fazer destas melhores práticas uma realidade, principalmente para as pequenas e médias empresas (PME's). Tendo-se em mente essas restrições e dada a sua importância para o processo de desenvolvimento de produtos, a proposta do presente trabalho é sistematizar essas melhores práticas e colocá-las à disposição da comunidade para acesso livre na internet, segundo a estrutura de um modelo de referência existente, proposto por Rozenfeld et al. (2006). Este modelo deve facilitar a sua aplicação. O livro, no qual o modelo foi descrito, contém somente a descrição do processo de forma holística, visando facilitar o seu entendimento pelo leitor, uma vez que ele é abrangente. As melhores práticas são descritas no livro dentro de caixas, pois existem métodos e ferramentas que podem ser utilizados em diversas atividades e normalmente não é simples para uma pequena/média empresa discernir a melhor forma de aplicá-las. O presente trabalho procura ir além do livro, colocando à disposição uma descrição mais detalhada das melhores práticas, com exemplos, roteiros detalhados para a sua aplicação, descrição de casos que possam motivar as empresas em adotá-las, e ferramentas de código livre para download gratuito.

Na Seção 2 é apresentada uma breve revisão bibliográfica, abordando os principais temas do trabalho: processo de negócio, mostrando a característica do PDP como um processo importante; o processo de desenvolvimento de produtos (PDP), que é o processo no qual as melhores práticas estão relacionadas; modelos de referência, pois as melhores práticas serão relacionadas a este modelo; e melhores práticas, que é o tema principal deste trabalho. Na seção 3 descreve-se a metodologia adotada no trabalho. $\mathrm{O}$ modelo de referência adotado para o PDP, é descrito de forma sucinta na seção 4, pois é a base para se estruturar as melhores práticas. Na seção 5 é apresentada a estrutura elaborada para a sistematização das melhores práticas. O do portal para a publicação das melhores práticas é mostrado na seção 6 . Na seção 7 discutem-se os resultados parciais obtidos. Em seguida são discutidas as conclusões e por fim as referências bibliográficas são listadas.

\section{Revisão bibliográfica}

\subsection{Processo de Negócio}

A visão por processos ultrapassa as barreiras físicas da organização e define uma forma de atuação, em que o objetivo principal é atender ao cliente. Todo produto ou serviço oferecido por uma empresa tem um processo associado a ele. Toda atividade desenvolvida em uma empresa faz parte de algum tipo de processo. Assim, na concepção mais geral, processo é qualquer atividade ou conjunto de atividades que utiliza um input, adiciona valor a ele e fornece um output a um cliente específico. Contudo, podemos avaliar o processo a partir de outros enfoques, como: fluxo de material, fluxo de trabalho, série de etapas, atividades coordenadas, mudança de estados. Uma discussão mais abrangente é encontrada em Gonçalves (2000).

Gonçalves (2000) divide os processos empresariais em três categorias: os processos de negócio; os processos organizacionais e os processos gerenciais. Esses processos são descritos na Figura 1.

Neste trabalho, aplica-se o conceito de processo de negócio, já que estamos descrevendo um fluxo de trabalho, um conjunto de atividades seqüenciadas, com inicio e fim definidos para o desenvolvimento de produto. Para permitir uma análise e estudo do desenvolvimento de produto, é fundamental caracterizá-lo como um processo de negócio. Isto porque, partindo desta abordagem, podem-se esclarecer as ligações críticas entre este processo e o mercado, conectando-o às necessidades dos clientes, e considerar as interações entre as habilidades e informações dos diversos setores funcionais de uma empresa, necessárias para o desenvolvimento de um produto (ROZENFELD, 1997; CLARK; FUJIMOTO, 1991). 


\begin{tabular}{|c|c|c|c|}
\hline Classe & Decrição & Tipo & Exemplo \\
\hline \multirow{2}{*}{$\begin{array}{lr}\text { Processo } & \text { de } \\
\text { Negócio } & \text { (de } \\
\text { cliente) } & \end{array}$} & \multirow{2}{*}{$\begin{array}{l}\text { São os processos que caracterizam a } \\
\text { atuação da empresa e que são } \\
\text { apoiados por outros processos } \\
\text { internos, resultando no produto ou } \\
\text { serviço que recebido por um cliente } \\
\text { externo. }\end{array}$} & $\begin{array}{l}\text { De } \\
\text { física }\end{array}$ & Fabricação de bicicletas. \\
\hline & & De serviço & $\begin{array}{l}\text { Atendimento de pedidos } \mathrm{de} \\
\text { clientes. }\end{array}$ \\
\hline \multirow{3}{*}{$\begin{array}{l}\text { Processo } \\
\text { organizacionais } \\
\text { (apoio aos } \\
\text { processos } \\
\text { produtivos) }\end{array}$} & \multirow{3}{*}{$\begin{array}{l}\text { São processos centralizados } \\
\text { na } \\
\text { organização e viabilizam o } \\
\text { funcionamento coordenado dos } \\
\text { vários subsistemas da organização } \\
\text { em busca de seu desempenho geral, } \\
\text { garantindo o suporte adequado aos } \\
\text { processos de negócio. }\end{array}$} & Burocráticos & Contas a pagar. \\
\hline & & Comportamentais & Integração empresarial. \\
\hline & & De mudança & $\begin{array}{l}\begin{array}{l}\text { Estruturação de } \\
\text { gerência. }\end{array}\end{array}$ \\
\hline \multirow[t]{3}{*}{$\begin{array}{l}\text { Processos } \\
\text { gerenciais }\end{array}$} & \multirow{3}{*}{$\begin{array}{l}\text { São processos focalizados nos } \\
\text { gerentes e nas suas relações e } \\
\text { incluem as ações de medição e ajuste } \\
\text { do desempenho da organização. }\end{array}$} & $\begin{array}{l}\text { De } \\
\text { direcionamento }\end{array}$ & Definição de metas da empresa. \\
\hline & & De negociação & 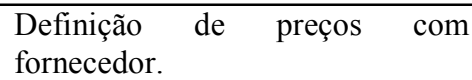 \\
\hline & & De monitoração & $\begin{array}{l}\text { Acompanhamento } \\
\text { planejamento e orçamento. }\end{array}$ \\
\hline
\end{tabular}

Figura 1 Classificação geral dos processos empresarias Fonte: GONÇALVES (2000)

Uma definição clássica e simples de processo de negócio é a apresentada por Davenport (1994) como "uma ordenação específica das atividades de trabalho no tempo e no espaço, com um começo, um fim, e inputs e outputs claramente identificados".

Processo de negócio pode ser entendido como um conjunto de atividades relacionadas entre si, que, quando executadas, transformam um determinado insumo em outro, (MOREIRA, 1994).

O conceito de processo de negócio é a base fundamental de todos os modelos de empresa. Ele substitui a clássica visão funcional por uma visão horizontal onde a unidade de análise passa a ser a cadeia de atividades/eventos existente dentro da empresa.

\subsection{O Processo de Desenvolvimento de Produto}

Desenvolvimento de produto é o processo em que "uma organização transforma dados sobre oportunidades de mercado e possibilidades técnicas em bens e informações para a fabricação de um produto comercial" (CLARK ; FUJIMOTO, 1991).

Clark e Fujimoto (1991) definem o processo de desenvolvimento de produto como um conjunto de atividades, em que a organização transforma dados sobre oportunidades de mercado e possibilidades técnicas em bens e informações para a fabricação de um produto comercial, abrangendo todas as áreas de uma empresa, desde as funções de marketing, engenharia de produto, produção. De forma complementar à definição de Clark e Fujimoto (1991), Wheelwright e Clark (1993) incluíram as atividades de planejamento estratégico e Rozenfeld et al. (2006) atividades de acompanhamento da produção e a descontinuidade do produto no mercado.

Clark e Fujimoto (1991) dividem esse processo em cinco etapas. Na proposta de Rozenfeld et al. (2006) o processo é descrito nas seguintes fases:

1. Planejamento estratégico de produtos;

2. Planejamento do projeto;

3. Projeto informacional;

4. Projeto conceitual; 
5. Projeto detalhado;

6. $\quad$ Preparação da produção do produto;

7. Lançamento do produto.

Adicionalmente às fases anteriores, os autores incluíram os processos de apoio, que são: 0 acompanhamento do produto e processo e a descontinuidade do produto.

Os textos clássicos de gestão de desenvolvimento de produto apresentam diferentes modelos de fases e etapas: Clark e Fujimoto, 1991; PUGH, 1991; Wheelwright e Clark, 1992; Cooper, 1993; Ulrich e Eppinger, 1995; Ullman, 1997; Baxter, 1998. O modelo de stage-gates proposto por Cooper (1993) descreve a introdução do produto na fábrica na fase denominada de teste e validação do produto. Wheelwright e Clark (1992) apresentam um modelo de fases para o PDP, o modelo de funil, que traz uma descrição da importância dos testes do processo produtivo, incluindo as atividades clássicas de produção piloto, definição de custos de fabricação, debugging, já em Clausing (1994) a ênfase é dada para a fase de preparação da produção.

Rozenfeld et al. (2006) procuram incorporar as características das propostas anteriores no seu modelo. Além disso, o modelo proposto destaca a integração com o planejamento estratégico da empresa juntamente com a gestão de portfolio; integra os conceitos do PMBOK (2002) na fase de planejamento de projeto, define ciclos integrados de detalhamento, aquisição e otimização dos produtos na fase de projeto detalhado; inclui atividades de otimização e validação do processo produtivo e técnicas direcionadas para a ergonomia e meio ambiente; propõe a fase de lançamento de produto integrada, na qual os processos de assistência técnica e vendas são desenhados e implementadas. O modelo proposto está integrado a dois outros processos de apoio: o de gerenciamento das mudanças de engenharia e melhoria do próprio PDP. Juntamente com o modelo os autores propõem um modelo de maturidade e um processo para a transformação do PDP.

Em bibliografia mais recente, Müller e Fairlie-Clarke (2003) elaboraram um método para auxiliar as empresas a avaliarem o PDP implantado ou em projeto. O método permite que a empresa utilize seus conhecimentos sobre seus produtos, processos, procedimentos e mercado relacionando ainda as melhores práticas ao gerenciamento do processo de desenvolvimento de novos produtos.

\subsection{Modelos de Referência}

O objetivo de um modelo de referência é prover a empresa de uma base, a partir da qual ela pode definir um modelo de processo particular (específico) da empresa. Podem ser desenvolvidos em situações reais ou em estudos teóricos, documentam os vários aspectos de um processo de negócio. Pode-se distinguir entre modelos procedimentais ou de implementação de softwares padrão, e modelos de negócios tais como modelos para gestão da produção e desenvolvimento de produtos (SCHEER, 1998). Além disso, os modelos de referência podem ser especializados para mercado, segmentos ou tipologias específicas, como por exemplo: setor automotivo, setor de alimentos, indústria aeroespacial e outros. Atualmente os principais fornecedores de modelos de referência são as empresas de consultoria e empresas de softwares corporativos. Essas empresas possuem modelos especializados para vários segmentos de mercado. Outro fornecedor de modelos de referência são os institutos de pesquisas e órgãos de pesquisa, porém os modelos tendem a ser mais genéricos e não tão detalhados.

Como já mencionado, o objetivo de construir modelos de referência é realizar uma descrição o mais abrangente possível de um determinado processo de negócio de forma que possa ser "customizada" para as diferentes situações, nas quais o processo é realizado. Sendo assim, o modelo deve agregar todas as atividades necessárias às diferentes instanciações do processo, as informações e recursos utilizados nessas atividades e a organização necessária a que sejam realizadas. Para Vernadat (1996) em um modelo de referência podem ser registradas as melhores práticas de um determinado processo.

Uma das formas de se representar a visão holística e caminhar no sentido da integração da manufatura é por meio da modelagem de empresa. A construção oferece aos profissionais das empresas uma 
referência para a discussão, estruturação e intervenção em casos específicos de forma a considerar todos os aspectos e elementos da situação. O modelo é ainda uma importante fonte de informação para a análise e introdução de novas ferramentas (tecnologias e metodologias) considerando os demais elementos da empresa.

Segundo Vernadat (1996), as vantagens em se adotar modelos de referência são:

- Redução de tempo e custo no desenvolvimento do modelo particular;

- Comparação das atividades da empresa com as atividades propostas no modelo (i.e., melhores práticas);

- Melhor suporte na implantação de sistemas de gestão empresarial integrados.

Um modelo pode ser utilizado como referência para a consolidação e implantação de vários business process, como também o processo de desenvolvimento de produto. Além disso, uma vez aplicado em uma determinada situação, ele irá constituir-se numa documentação do processo de desenvolvimento e num importante dispositivo para guiar intervenções futuras dentro deste processo (na Figura 2 resumem-se os principais aplicações do modelo de referência).

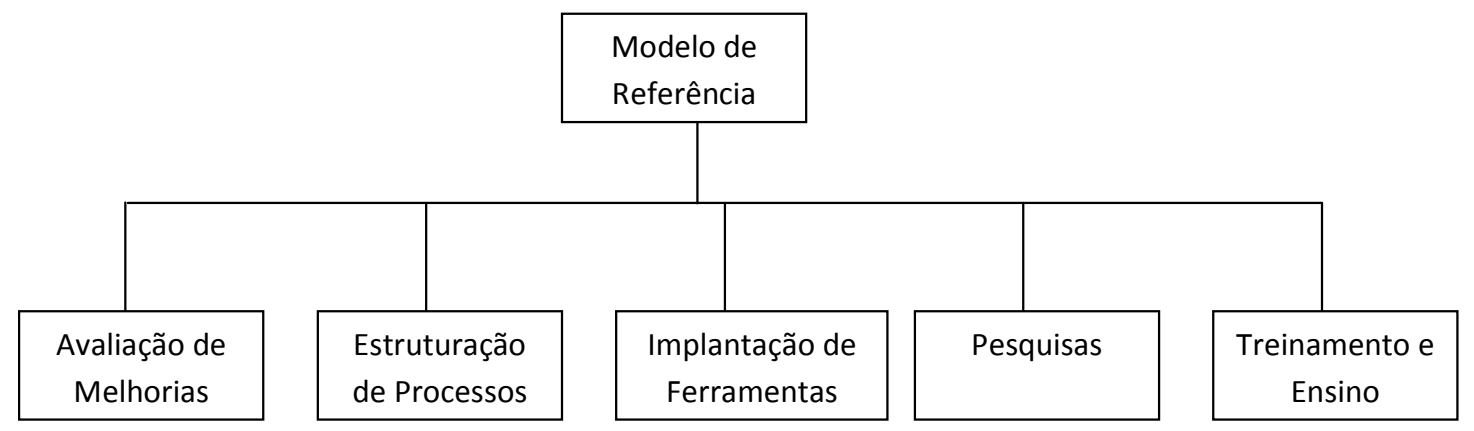

Figura 2 Principais Usos de um Modelo de Referência

Kalpic e Bernus (2002) que apresentam um modelo para melhorar o processo de desenvolvimento de novos produtos e gerenciar conhecimentos.

Muffatto (1998) realizou um estudo dos modelos propostos por empresas japonesas para o processo de desenvolvimento de produtos, focando as características da estratégia baseada na variedade e aponta algumas direções de novos modelos de desenvolvimento que devem incluir os aspectos do lean design e o desempenho de multi-projetos.

\subsection{Melhores práticas do PDP}

O crescimento de uma organização depende que exista flexibilidade no processo de negócio de modo a se adaptar às novas necessidades de seus clientes. $\mathrm{O}$ gerenciamento de informações e conhecimentos no processo de desenvolvimento de produto tem importante papel nesse cenário, pois permite agilidade no tratamento das informações. A forma como a organização utiliza seus recursos e as variedades de recursos disponíveis determinam o diferencial para o seu desempenho. Assim, nesse contexto a informação é claramente um recurso necessário para o PDP. Zahay et al. (2004) reforçam esse fato, apresentando um estudo exploratório que investiga a importância do gerenciamento do conhecimento e da informação para o PDP.

Sánchez e Pérez (2003) apresentam um survey que investiga o relacionamento das melhores práticas individuais e as atividades de cooperação no processo de desenvolvimento de novos produtos e sua complexidade tecnológica. Os autores utilizam as práticas identificadas por Millson et al. (1992) e por Dröge et al. (2000) e adicionaram novas técnicas tais como a prototipagem rápida e as associaram ao tamanho das empresas. Uma das conclusões do artigo foi mostrar que algumas práticas estão 
associadas positivamente com o tamanho da empresa, por exemplo: $\mathrm{CAD} / \mathrm{CAE}$, engenharia simultânea, times multifuncionais para inovação, desenvolvimento de fornecedor. Os autores indicam que as companhias com maior portfolio de produtos tecnologicamente complexos terão maior desvantagem que as companhias com produtos menos complexos, sugerindo que pequenas empresas não estão em desvantagem no desenvolvimento de produto.

Um modelo de referência pode ser constituído por uma coleção de melhores práticas relacionadas com o processo representado (Rozenfeld et al. (2006); Chrissis et al. (2003)). Então a sistematização das melhores práticas de PDP inclui a sua representação em um modelo de referência.

Pelas referências bibliográficas apresentadas, pôde entender a importância das melhores práticas de PDP registradas em modelos de referência.

\section{Metodologia}

A proposta deste trabalho tem natureza aplicada, já que tem como objetivo gerar conhecimentos dirigidos à solução de um problema específico, que no caso pode ser definido como: falta uma sistemática para a utilização das melhores práticas no processo de desenvolvimento de produto. Em relação aos objetivos, a pesquisa pode ser classificada como pesquisa-ação, pois se constitui principalmente de uma proposta, que visa elaborar uma estrutura para permitir a utilização das melhores práticas do PDP que hipoteticamente contribuirão para viabilizar a utilização de modelos de referência por PME's. Isso será desenvolvido com base no proposto por Rozenfeld et al. (2006). A abordagem é qualitativa, uma vez que o foco do trabalho é no processo e em seu significado, Gil, (1991).

A sistemática de desenvolvimento do trabalho inclui as seguintes etapas:

1. Pesquisa bibliográfica para consolidação das melhores práticas em PDP;

2. Sistematizar as melhores práticas no PDP;

3. Elaborar da estrutura para representar as melhores práticas, de modo que seja suficientemente informativa para sua utilização;

4. Projetar um portal para o modelo unificado proposto por Rozenfeld et al. (2006);

5. Publicar as melhores práticas na web por meio desse portal;

6. Testes e avaliação.

\section{O Modelo unificado para o processo de desenvolvimento de produtos o}

O modelo unificado para o processo de desenvolvimento de produtos, proposto por Rozenfeld et al. (2006) contém os conceitos e melhores práticas em desenvolvimento de produto (DP) e foi desenvolvido a partir de conhecimentos compartilhados por uma rede de pesquisadores brasileiros que sintetizou a experiência de três grupos de pesquisa sobre gestão do desenvolvimento de produtos. $\mathrm{O}$ modelo unificado é composto por três partes descritas a seguir.

1. Modelo de referência do PDP: descreve as melhores práticas para a gestão do processo de desenvolvimento de produto, relacionando técnicas e métodos às atividades do processo. $\mathrm{O}$ modelo de referência processo foi divido em 3 macro-fases: Pré-Desenvolvimento, Desenvolvimento e PósDesenvolvimento. Cada uma das macro-fases foi dividida respectivamente em fases, atividades e tarefas que juntas traduzem as melhores práticas para o PDP. Existem atividades que se repetem em todas as fases do desenvolvimento, como a atividade "avaliar fase" (gates), "monitoramento da viabilidade econômico-financeira" e "documentação das decisões tomadas e lições aprendidas". No modelo, elas são agrupadas sob o rótulo de atividades genéricas. A macro-fase de prédesenvolvimento é o elo entre as estratégias da empresa e a definição dos projetos de desenvolvimento de produtos. Nessa macro-fase agrupam-se duas fases: Planejamento Estratégico de produtos e o Planejamento do projeto, envolvendo o gerenciamento de portfolio de projetos e avaliações dos projetos escolhidos, verificando se esses devem ser continuados ou não. A macro-fase de desenvolvimento agrupa as fases de projeto informacional, projeto conceitual, projeto detalhado, preparação para produção e lançamento do produto. Todas essas fases visam um detalhamento das informações técnicas, comerciais e de produção, envolvendo elementos como desenhos técnicos, 
protótipos, homologações, registros, parcerias com fornecedores e processos de produção. A macrofase de pós-desenvolvimento agrupa a fase de acompanhar produto e processo e a fase de descontinuar o produto. O acompanhamento do ciclo de vida do produto, a avaliação do seu desempenho no mercado, sua retirada e quais processos de melhoria podem ser implementados são feitos nessa macro-fase. A figura 2 mostra uma visão geral desse modelo.

2. Modelo de maturidade do PDP: tem a função de apoiar a identificação do nível de evolução que a empresa se encontra. Ele descreve níveis de maturidade e apresenta quais atividades devem estar formalizadas e implementadas em cada um destes níveis. A descrição mostra, portanto, uma hierarquia de prioridade em termos de atividade à medida que os níveis mais altos são atingidos uma vez que os níveis anteriores tenham sido satisfeitos;

3. Modelo de transformação do PDP: descreve um processo estrutural para implementar as mudanças necessárias no PDP, visando a elevação do nível de maturidade, por meio de projetos de transformação.

A partir das melhores práticas apontadas no modelo de referência, utiliza-se o modelo de transformação para atingir um determinado nível de maturidade. Esse relacionamento é ilustrado na Figura 3.

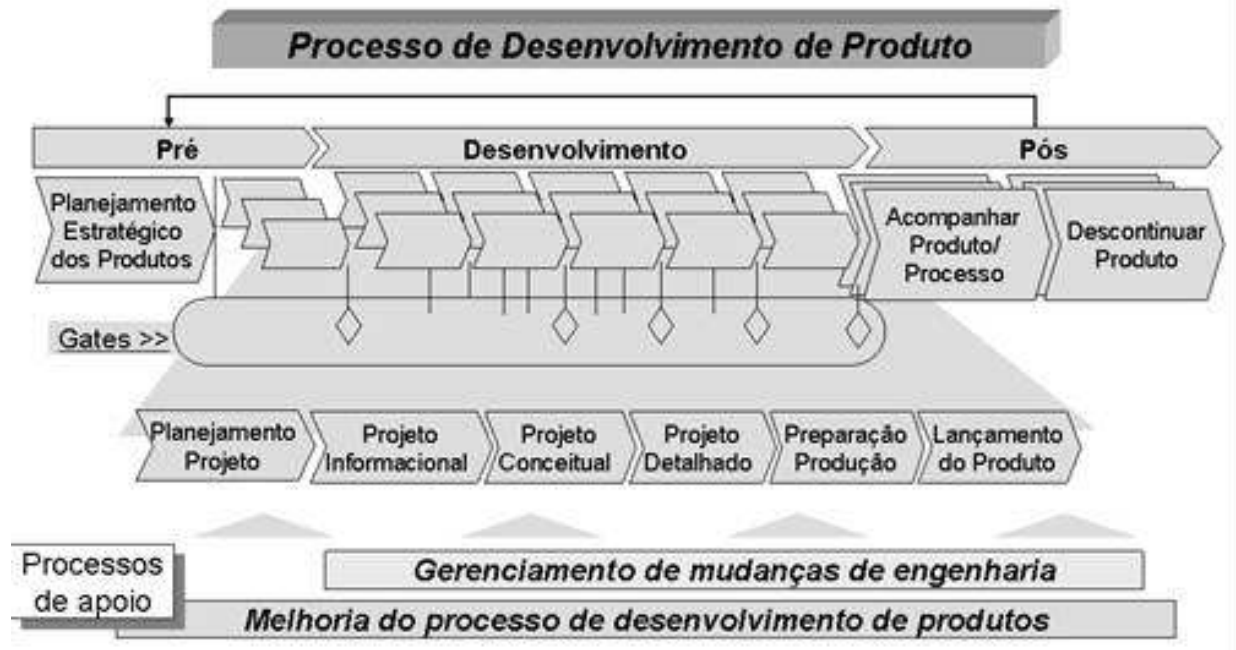

Figura 3 Modelo unificado para o processo de desenvolvimento de produto, ROZENFELD et al. (2006)

\section{Sistematização das melhores práticas do modelo unificado}

O modelo unificado para a gestão do processo de desenvolvimento de produtos, proposto por Rozenfeld et al. (2006) organiza as atividades do PDP em fases e reúne um conjunto de informações, métodos e ferramentas úteis para auxiliar o processo. A esse conjunto estamos chamando de melhores práticas. Com a finalidade de sistematizar esses conteúdos elaboramos uma classificação para estruturar o processo de busca e organização. Essa classificação permite que se encontre uma melhor prática a partir:

- Da fase / atividade a que ela se relaciona;

- Da entrega (deliverable) resultante da sua aplicação;

- Da tecnologia relacionada a ela;

- Do tipo de produto ou setor produtivo, para o qual ela é voltada;

- E de sua natureza, conforme apresentado a seguir: 
1. Metodologia: é um conjunto de métodos

2. Método: é um conjunto de princípios, regras, organizados para a realização de uma ou mais atividade;

3. Aplicativo: é um conjunto de ferramentas;

4. Ferramenta: é a automatização total ou parcial de um ou mais métodos ou atividades;

5. Funcionalidade: é uma automação específica dentro de um aplicativo ou ferramenta que corresponde a transformação de um input em um output;

6. Informação: dados ou documentos de entradas e saídas das atividades.

Esses elementos são utilizados para apoiar a realização das atividades do PDP e fornecem uma base para a consolidação das atividades. Definimos um padrão para a publicação de conteúdos, descritos na Figura 5.

Essa proposta de estrutura para descrever as melhores práticas torna-se mais rica quando associada aos recursos disponíveis na web, pois podemos fornecer um aspecto dinâmico ao processo, ou seja, esses conteúdos podem ser constantemente melhorados a medida que novos exemplos forem incluídos, ou outros roteiros sejam sugeridos. Na proposta do trabalho, pretende-se desenvolver um portal de livre acesso e compartilhamento, respeitando-se critérios de acesso (perfil de cadastramento) para garantir que somente informações válidas sejam incluídas no portal.

A estrutura apresentada na Figura 5 representa uma parte das melhores práticas, que será estendida para as demais.

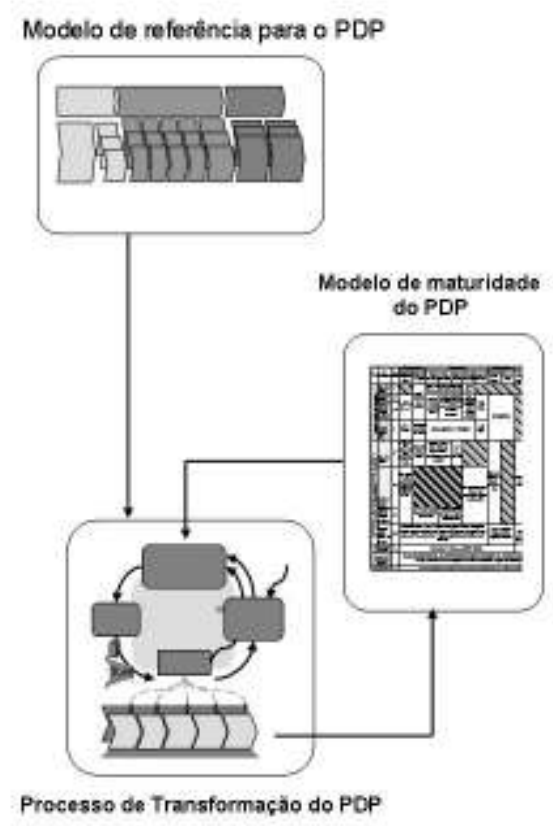

Figura 4 Componentes do modelo unificado do PDP

\section{Portal PDPnet}

Existe uma proposta similar à proposta deste trabalho realizada pelo Massachusetts Institute of Technology - MIT (acessado pela URL http://ccs.mit.edu/ph/). A proposta do projeto do Process Handbook tem as metas divididas por usuários:

- Para um pesquisador ou educador: o usuário poderia sistematizar uma grande quantidade de elementos que se conhece sobre o negócio, por exemplo: princípios básicos, os resultados mais 
importantes de uma pesquisa, exemplos úteis de casos. Esses conhecimentos poderiam ser criados e compartilhados eletronicamente com outros pesquisadores, educadores e estudantes de todo o mundo. Essa estrutura tem o objetivo de ajudar os usuários a rapidamente encontrar coisas necessárias para organizar idéias sobre a organização.

Figura 5 Detalhamento da classificação das melhores práticas segundo a sua natureza de apoio ao PDP

\begin{tabular}{|c|c|c|}
\hline Conteúdo & Tipo & Descrição \\
\hline \multirow{7}{*}{ Metodologia } & Descrição & $\begin{array}{l}\text { Descrição sucinta da metodologia com seu objetivo e suas principais } \\
\text { características. }\end{array}$ \\
\hline & Roteiro & $\begin{array}{l}\text { Descrição de uma seqüência de passos básicos para a aplicação da } \\
\text { metodologia. }\end{array}$ \\
\hline & Padrões & São documentos que auxiliam a utilização do roteiro. \\
\hline & Exemplo & Descrição detalhadamente uma aplicação da metodologia. \\
\hline & Referência & $\begin{array}{l}\text { Um conjunto de informações adicionais, complementares relacionadas a } \\
\text { metodologia, por exemplo: especialistas, associações, artigos, livros. }\end{array}$ \\
\hline & Links & Sites relacionados a metodologia. \\
\hline & $\begin{array}{l}\text { Aplicação da } \\
\text { metodologia no } \\
\text { modelo de DP }\end{array}$ & Lista as atividades em que a metodologia é utilizada no modelo de DP. \\
\hline \multirow{7}{*}{ Método } & Descrição & $\begin{array}{l}\text { Descrição sucinta do método com seu objetivo e suas principais } \\
\text { características. }\end{array}$ \\
\hline & Roteiro & Descrição de uma seqüência de passos básicos para a aplicação do método. \\
\hline & Padrões & São documentos que auxiliam a utilização do roteiro. \\
\hline & Exemplos & Descrição detalhadamente uma aplicação do método. \\
\hline & Referências & $\begin{array}{l}\text { Um conjunto de informações adicionais, complementares relacionadas ao } \\
\text { método, por exemplo: especialistas, associações, artigos, livros. }\end{array}$ \\
\hline & Links & Sites relacionados ao método. \\
\hline & $\begin{array}{l}\text { Aplicação no } \\
\text { modelo de DP }\end{array}$ & Lista as atividades em que o método é utilizado no modelo de DP. \\
\hline \multirow{5}{*}{ Aplicativo } & Descrição & $\begin{array}{l}\begin{array}{l}\text { Descrição sucinta do aplicativo com seu objetivo e suas principais } \\
\text { características. }\end{array} \\
\end{array}$ \\
\hline & Tutorial & Descrição e explicação de como utilizar as funcionalidades do aplicativo. \\
\hline & Exemplo & Descrição detalhadamente da utilização do aplicativo. \\
\hline & Links & Sites relacionados o aplicativo. \\
\hline & $\begin{array}{l}\text { Aplicação no } \\
\text { modelo de DP }\end{array}$ & Lista as atividades em que o aplicativo é utilizado no modelo de DP. \\
\hline \multirow{6}{*}{ Ferramenta } & Descrição & $\begin{array}{l}\text { Descrição sucinta da ferramenta com seu objetivo e suas principais } \\
\text { características. }\end{array}$ \\
\hline & Tutorial & Descrição e explicação de como utilizar as funcionalidades do aplicativo. \\
\hline & Exemplos & Descrição detalhadamente uma aplicação da ferramenta. \\
\hline & Referências & $\begin{array}{l}\text { Um conjunto de informações adicionais, complementares relacionadas com } \\
\text { a ferramenta, por exemplo: tutoriais, especialistas, associações, artigos, } \\
\text { livros. }\end{array}$ \\
\hline & Links & Sites relacionados a ferramenta. \\
\hline & $\begin{array}{l}\text { Aplicação no } \\
\text { modelo de DP }\end{array}$ & Lista as atividades em que a ferramenta é utilizada no modelo de DP. \\
\hline \multirow[t]{4}{*}{ Funcionalidade } & Descrição & $\begin{array}{l}\text { Descrição sucinta da funcionalidade com seu objetivo e suas principais } \\
\text { características. }\end{array}$ \\
\hline & Tutorial & Descrição e explicação de como utilizar a funcionalidade. \\
\hline & Entrada & Dados necessários para a utilização da funcionalidade. \\
\hline & Saída & Dado de resposta fornecido pela funcionalidade. \\
\hline \multirow{4}{*}{ Informação } & Descrição & Descrição sucinta da informação. \\
\hline & Padrão & Documentos que auxiliam a utilização da informação. \\
\hline & Exemplo & Exemplo de um padrão. \\
\hline & Fluxo & Representa o fluxo da informação no modelo de DP. \\
\hline
\end{tabular}

- Para um gerente ou consultor: o usuário poderia utilizar os conhecimentos sobre as melhores práticas, casos e softwares do mundo todo. O usuário poderia criar sua própria versão para esses 
conhecimentos e compartilhar detalhes de informações sobre pontos importantes das atividades de sua empresa ou clientes, por exemplo: o que precisa ser feito, quem é o responsável e quais os recursos disponíveis.

Algumas dessas funcionalidades estão implementadas no portal, mas esses trabalham continuam, numa visão de que essas idéias são viáveis e necessárias.

A estrutura que propomos tem uma versão inicial, que foi denominada PDPnet (http://www.pdp.org.br). Sua estrutura segue fundamentalmente o modelo de referência proposto em Rozenfeld et al. (2006). Neste trabalho tentamos fornecer aos usuários novas dimensões de consulta, similares ao que foi proposto MIT Project Handbook, já que a internet dispõe de uma grande gama de recursos que viabiliza novos canais de aprendizado, podem ser por exemplo recursos de visualização gráfica, download de documentos, links para páginas associadas e a própria dinâmica da navegação pelo conteúdo do portal.

O portal permite acesso gratuito e disponibiliza as melhores práticas organizadas conforme a Figura 5. A busca das melhores práticas pode também ser realizada por meio da classificação inicial descrita. $\mathrm{O}$ livre acesso possibilita que empresas ou qualquer tipo de usuário tenham acesso a esse conteúdo sem que para isso exista a necessidade de qualquer tipo de investimento adicional ao mínimo acesso à internet. O modelo poderá ser consultado sem restrições para que, principalmente as PME's possam utilizá-lo como guia em suas atividades de gestão do processo de desenvolvimento de produtos.

Caso a empresa tenha interesse será possível instanciá-lo, para que algumas adaptações sejam feitas para melhor representar as atividades da empresa, já que o que estamos propondo é um modelo genérico. Em outras palavras, a empresa pode "copiar" o modelo de referência e depois descartar tudo aquilo que não é apropriado para a sua realidade, ou para o seu nível de maturidade. Como o interesse é de agregar conhecimento e experiências associadas ao PDP, todos os novos conhecimentos associados a comentários, novos exemplos etc. podem ser inseridos, mediante uma aprovação, para que os conhecimentos publicados sejam cofiáveis. Tem-se então um ambiente interativo com o usuário e um portal que compartilha conhecimentos continuamente novos.

No projeto navegacional do portal elaboramos diversas formas de visualização do modelo e de seu conteúdo Figura 6, que são:

1. Visão gráfica: nesta opção o usuário visualiza o modelo graficamente de duas maneiras: as macro-fases e suas respectivas fases, fases e suas respectivas atividades;

2. Mapa do modelo: nesta opção o usuário acessa o modelo a partir de uma estrutura em árvore, com dois subníveis ( fase, atividade);

3. Menu: nesta opção o usuário navega pelo modelo a partir de um menu lateralmente localizado, que descreve todas as macro-fases e fases do modelo unificado. É possível percorrer por todas as fases do modelo e acessar suas respectivas atividades.

O portal é composto basicamente por quatro tipos de páginas:

- Um tipo para as fases do modelo, onde estão listadas as atividades associadas à fase e todas as informações de entrada e saída de cada uma delas, os critérios do gate e as entregas;

- Uma página para as atividades onde estão descritas as tarefas associadas à atividade, as informações de entrada e saída e ainda quais os métodos/ferramentas úteis para a realização da atividade;

- Uma página para descrever os métodos/ferramentas, organizada de acordo com a estrutura proposta na Figura 5;

- E uma página para representar as informações, que contém a descrição da informação, os padrões, exemplos e o fluxo da informação vide Figura 6. 


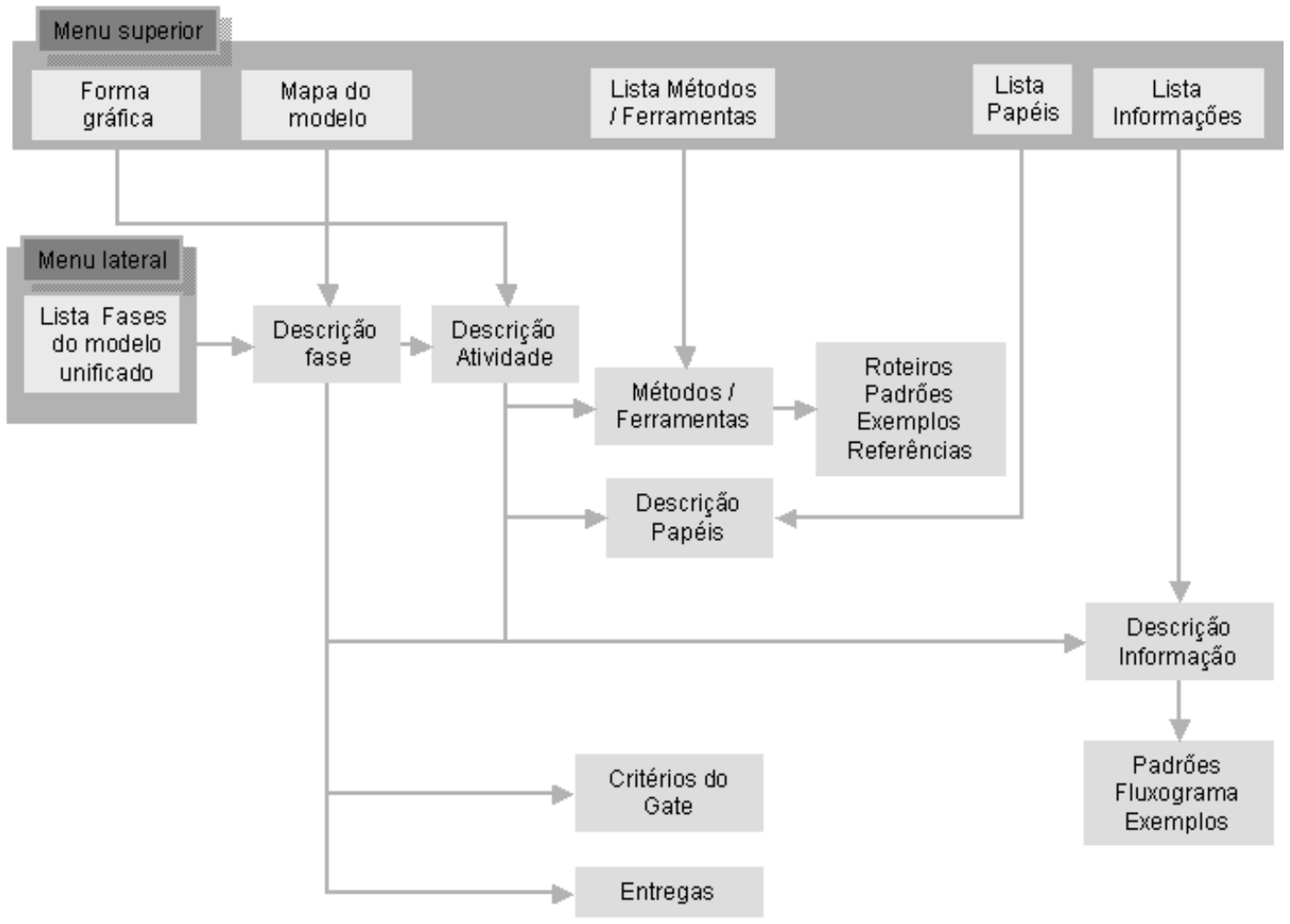

Figura 6 Estrutura do portal

Para acessar diretamente uma informação de entrada/saída das atividades ou algum tipo de ferramenta/método existe uma opção no menu superior Figura 6. A partir dela o usuário acessa uma lista em ordem alfabética das informações ou das ferramentas/métodos. A Figura 7 descreve um exemplo de página de fase (no caso a fase 3, o projeto informacional). Este ilustra uma consulta ao Modelo, representado pelo número 1, fase 3 (Projeto informacional), dentro da macro-fase "Desenvolvimento". A página da fase mostra todas as atividades, informações de entrada/saída, a opção de visualizar essas informações graficamente. Cada atividade possui um link para a página referente às atividades. Na página de atividades Figura 8 estão listadas todas as tarefas relacionadas à atividade assim como as informações de entradas/saídas e os métodos/ferramentas úteis para sua realização. Existe um link nas ferramentas/métodos para a sua respectiva página, ilustrada na Figura 9. Cada informação de entrada/saída possui um link para a página referente à informação, ilustrada na Figura 10.

A definição do conteúdo inicial inserida no portal, na forma de detalhamento do modelo de referência foi baseada nas necessidades das PME percebidas pela equipe de desenvolvimento do portal. 


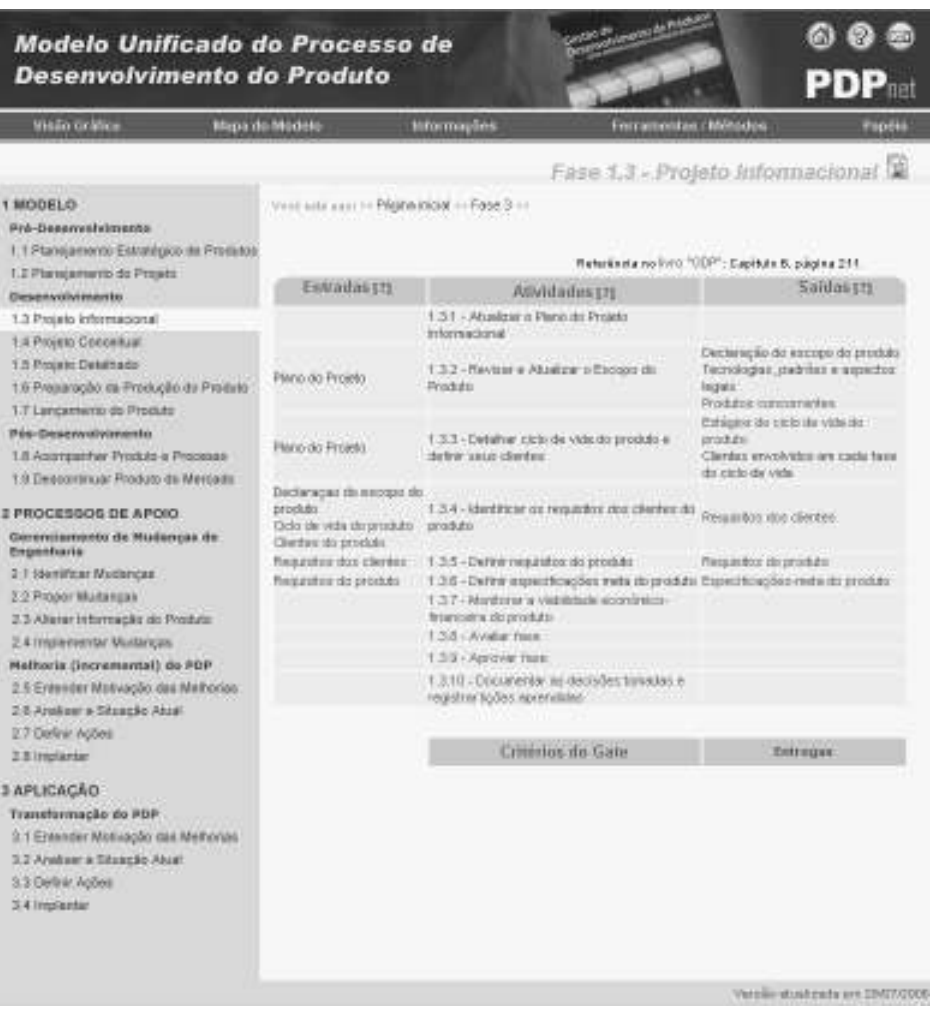

Figura 7 Página do portal PDPnet de descrição de fase

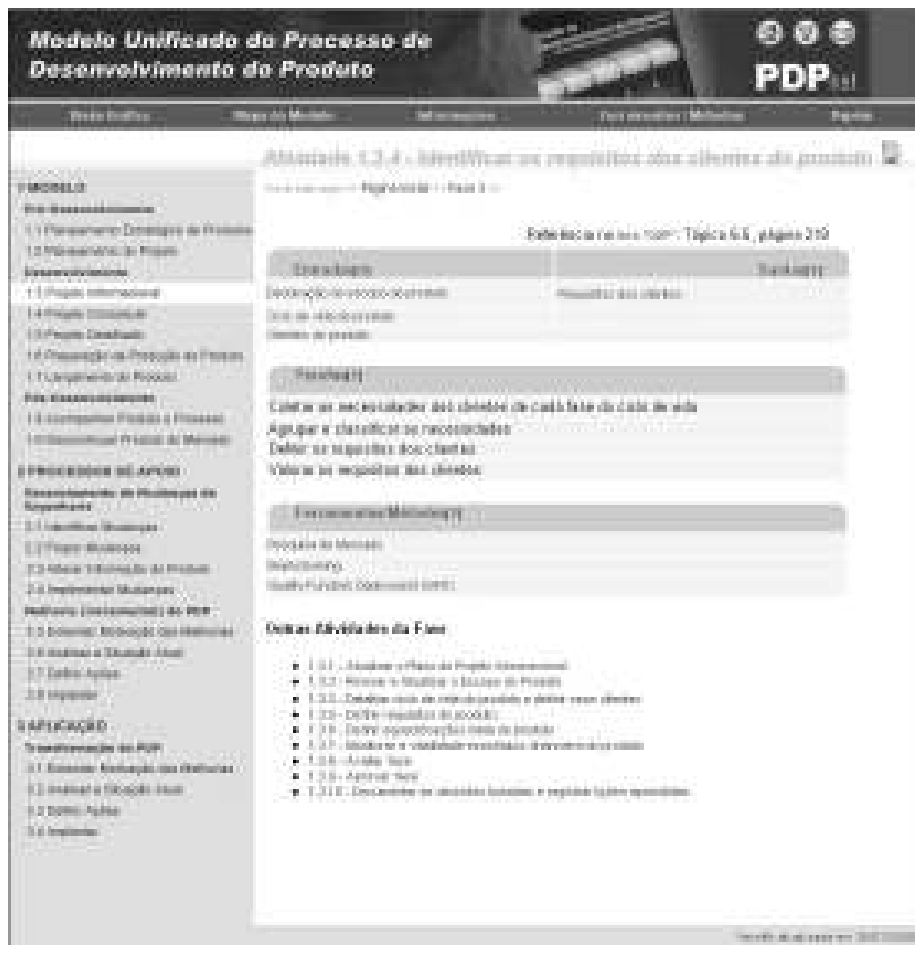

Figura 8 Página do portal PDPnet de descrição de atividades 


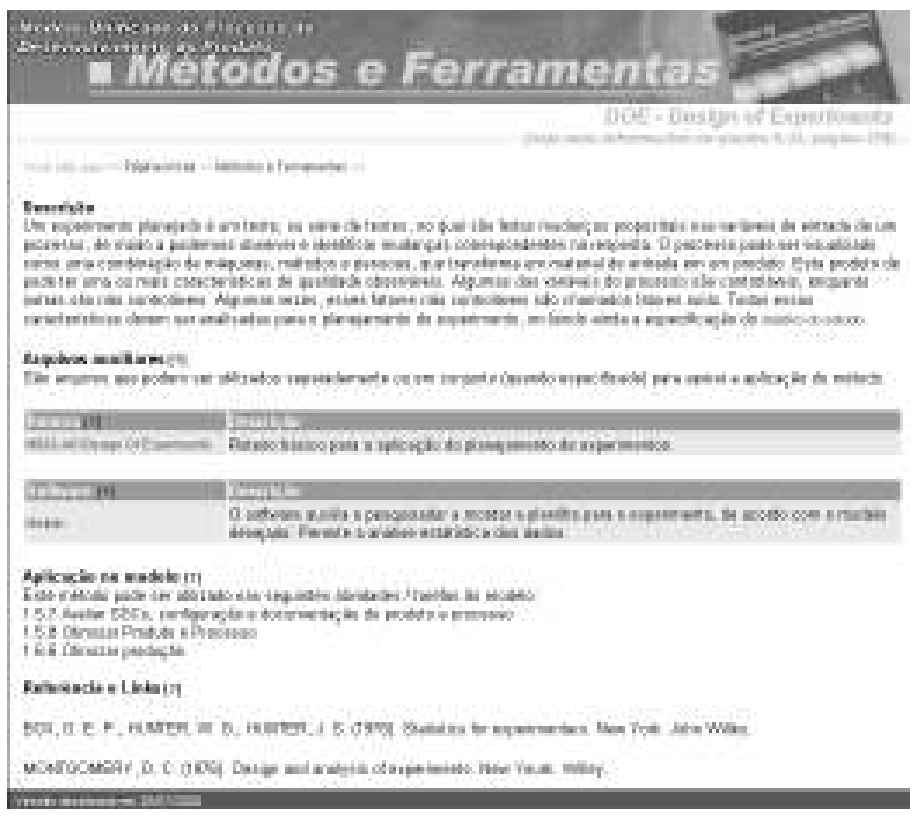

Figura 9 Página do portal PDPnet de descrição de métodos/ferramentas

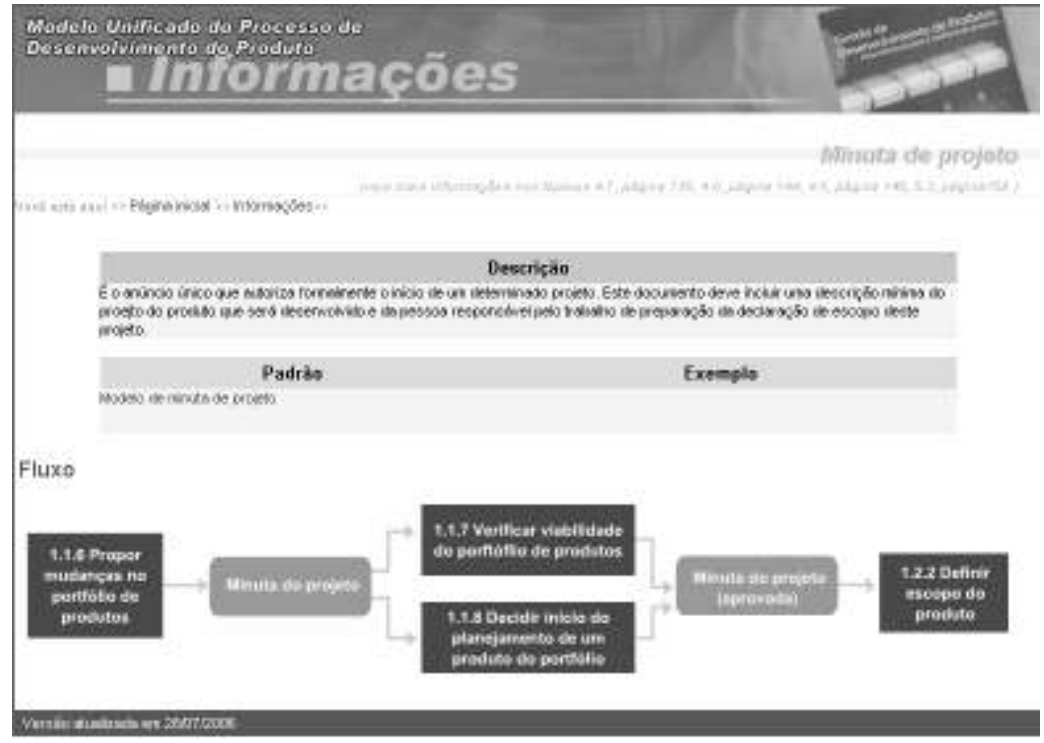

Figura 10 Página do portal PDPnet de descrição de informações

\section{Resultados}

A estrutura proposta para representar as melhores práticas do PDP, de acordo com Rozenfeld et al. (2006) foi implementada em um portal denominado PDPnet. Esse portal encontra-se disponível para acesso por meio da URL http://www.pdp.org.br. Embora o site não tenha sido divulgado amplamente, pode ser encontrado por meio de buscadores. Assim temos um histórico de acessos. Essas informações de acesso representam os primeiro resultados para a avaliação da proposta. Na verdade, é um indicativo de interesse no assunto, sem o compromisso de medir o impacto dessas informações. Contudo, a descrição de acessos fornece de certa maneira caminhos para a melhoria do portal, tanto em termos de conteúdos como de usabilidade. Em particular, as questões relacionadas com a usabilidade do portal devem ser abordadas com maior profundidade, prevendo a elaboração de teste, já que é a forma de medir como o portal está sendo compreendido pelos usuários. Os dados apresentados 
nesse trabalho foram coletados no dia 22 de agosto de 2006.

Analisando a Tabela 1, podemos identificar um grande potencial para o portal, já que o protótipo tem em média 150 visitas por dia, sendo que esses visitantes acessam em média 6 páginas do portal, por um tempo médio de 7 minutos. Esses dados também indicam que apesar do interesse pelo assunto é necessário o aprimoramento no conteúdo do portal, fator que depende principalmente de tempo para a elaboração e padronização das informações em formato para a publicação na web.

A Tabela 2 mostra as páginas mais visitadas. Essas visitas sugerem um maior interesse dos usuários pela fase de planejamento do projeto dentro do modelo de referência. A Tabela 2 mostra a forma como o usuário acessa o portal, ou seja, qual o assunto que o levou até o portal. Cerca de $20 \%$ acessam o portal a partir da página inicial, ou seja, seu interesse foi pelo assunto "desenvolvimento de produto". Em segundo lugar está o assunto 'Planejamento de projeto', indicando novamente que essas informações despertam o interesse do usuário.

Tabela 1 Estatísticas gerais de acesso ao portal PDPnet até o dia 22 de agosto de 2006.

\begin{tabular}{|l|l||l||}
\hline \multicolumn{2}{|l|}{ Estatísticas Gerais } & 69.983 \\
\hline \hline \multirow{4}{*}{ Páginas visitadas } & Páginas visitadas & 442 \\
\cline { 2 - 3 } & Média por dia & 6 \\
\cline { 2 - 3 } & Média por único visitante & 55.051 \\
\cline { 2 - 3 } & Documentos visitados & 23.768 \\
\hline \hline \multirow{2}{*}{ Visitas } & Visitas & 150 \\
\cline { 2 - 3 } & Média por dia & $00: 07: 23$ \\
\cline { 2 - 3 } & Tempo médio da visita & $00: 02: 31$ \\
\cline { 2 - 3 } & Tempo mediano da visita & 10.132 \\
\hline \hline \multirow{2}{*}{ Visitantes } & Único visitante & 7.723 \\
\cline { 2 - 3 } & Visitantes de uma visita & 2.409 \\
\cline { 2 - 3 } & Visitantes de mais de uma \\
& visita & \\
\hline \hline
\end{tabular}

Tabela 2 Páginas visitadas até o dia 22 de agosto de 2006.

\begin{tabular}{|c|c|c|c|}
\hline & Páginas & Visitas & $\begin{array}{c}\text { Tempo } \\
\text { médio } \\
\text { da visita }\end{array}$ \\
\hline 1 & $\begin{array}{l}\text { Desenvolvimento de Produto } \\
\text { http://www.pdp.org.br/ }\end{array}$ & 3.995 & 00:02:20 \\
\hline 2 & $\begin{array}{l}\text { Modelo de Referência - Planejamento do Projeto } \\
\text { http://www.pdp.org.br/ ModeloLivroWeb/www/fase } 2 . h t m\end{array}$ & 1.778 & 00:01:10 \\
\hline 3 & $\begin{array}{l}\text { Modelo de Referência - Planejamento Estratégico de Produtos } \\
\text { http://www.pdp.org.br/ ModeloLivroWeb/www/fase1.htm }\end{array}$ & $\mid 1.312$ & 00:01:02 \\
\hline 4 & $\begin{array}{l}\text { Comunidade } \\
\text { http://www.pdp.org.br/phpbb/ profile.php }\end{array}$ & 906 & 00:00:43 \\
\hline 5 & $\begin{array}{l}\text { Mapa do Modelo } \\
\text { http://www.pdp.org.br/ ModeloLivroWeb/www/mapa.htm }\end{array}$ & 855 & 00:01:13 \\
\hline
\end{tabular}




\section{Conclusões}

De modo geral podemos concluir que existe grande interesse no assunto PDP e que temos um importante papel para contribuir na elaboração e difusão desses conhecimentos. Percebemos o grande potencial existente e que a demanda por esse tipo de informação tende a crescer. Pela avaliação dos acessos, existe interesse pelos documentos disponíveis das páginas. Este trabalho está dentro da tendência de se oferecer cada vez mais conteúdo de forma livre na internet.

A definição do conteúdo que deve ser detalhado com profundidade no portal deve ser ainda direcionada pelas PMEs e para isso deve-se realizar um trabalho de levantamento mais abrangente. Por enquanto, utiliza-se a experiência da equipe de desenvolvimento do portal, que possui anos de experiências em trabalhos práticos de melhoria do PDP e na especificação de modelos de referência. Isso pode ser comprovado pelo próprio livro que descreve de maneira holística o modelo, descrevendo as fases do PDP.

A análise dos dados preliminares sobre a utilização do portal indicou ainda que é preciso mais do que apenas disponibilizar a informação para acesso na web para que sejam utilizadas ou que contribuam para a melhoria do PDP das empresas ou para o aprendizado de outros usuários. Melhorar a usabilidade é outra questão importante, pois permite que o portal seja utilizado sem complicações, ou no mínimo de forma lógica e fácil e com certeza, melhorar o conteúdo do portal para torná-lo sempre atrativo. Por não existir nada similar disponível na web, a proposta é ainda mais necessária.

Como próximos passos, devem-se adicionar outras melhores práticas no modelo de referência para em seguida partir para avaliação de seu uso pelas PME, por meio de estudos de caso, analisando se o conteúdo e a estrutura são adequados às suas necessidades ou expectativas.

\section{Referências}

BAXTER, M. Projeto de produto: guia prático para o desenvolvimento de novos produtos. São Paulo: Edgard Blücher, 1998.

CLARK, K. B.; WHEELWRIGHT, S. C. Managing new product and process development: texts and cases. New York: Free press, 1993.

CLARK, K.B.; FUJIMOTO, T. Product development performance: strategy, organization and management in the world auto industry. Boston-Mass., Harvard Business School Press, 1991.

CLAUSING, D. Total quality development. New York: ASME Press, 1994.

DAVENPORT, T. H. Reengenharia de Processos. Rio de Janeiro: Campus, 1994.

GARVIN, D.A. The processes of organization and management. Sloan management review, v. 39, n. 4, p.33-50, 1998.

GIL, A. C. Como elaborar projetos de pesquisa. 3ed., São Paulo: Atlas, 1991.

GONÇALVES, J. E. L. As empresas são grandes coleções de processos. RAE, v. 40, n. 1, p. 6-19, 2000.

KALPIC, B. \& BERNUS, P. Business process modelling in industry - the powerful tool in enterprise management. Computers in Industry, v. 47, p. 299-318, 2002.

MUFFATTO, M. Reorganizing for product development: evidence from Japanese automobile firms. International Journal of Production Economics, v. 56-57, p. 483-493, 1998. 
MÜLLER, M. H. \& FAIRLIE-CLARKE, A.C. The evaluation fo manufacturing issues in the product development process. Journal of Materials Processign Technology, n 138, p. 2-8, 2003.

PROJECT MANAGEMENT INSTITUTE. A guide to the project management body of knowledge (PMBOK guide), Pennsylvania, 2002.

PUGH, S. Total design: integrated methods for successful product engineering. Reading, HA: Addison, 1978.

ROZENFELD, H. Reflexões sobre a Manufatura Integrada Por Computador (CIM), Proceedings of Manufatura Classe Mundial: Mitos e Realidades. São Paulo, 1997.

MOREIRA, D. A. Reengenharia : dinânica para a mudança. São Paulo: Livraria Pioneira Editora, 1994.

ROZENFELD, H. et al. Gestão de Desenvolvimento de Produtos - uma referência para a melhoria do processo. São Paulo: Saraiva, 2006. 542p

SÁNCHEZ, A. M. \& PÉREZ, M. P. Flexibility in new product development: a survey of practices and its relationship with the product's technological complexity. Technovation, v. 23,p. 139-145, 2003.

SMITH, R. P. \& MORROW, J. A. Product development process modeling. Design studies, v. 20, p. 237-261, 1999.

ULLMAN, D. G. The mechanical design process. New York: McGraw-Hill International Editions, 1997.

ULRICH, K. T.; EPPINGER, S. D. Product design and development. New York: McGraw-Hill, 1995.

VERNADAT, F.B. Enterprise Modeling and Integration: Principles and Applications. London: Chapman \& Hall., 1996.

WHEELWRIGHT, S.C.; CLARK, K.B. Revolutionizing Product Development: quantum leaps in speed, efficiency, and quality. New York: The Free Press, 1992.

ZAHAY, D. GRIFFIN, A. FREDERICKS, E. Sources, uses, and forms of data in the new product development process. Industrial Marketing Management, 33, p. 657-666, 2004.

WAGNER, E. L. et al. The creation of "best practice"software: myth, reality and ethics. Information and organization, 16, p. 251-275, 2006. 